\title{
La cláusula de no-discriminación en la Decisión 578: ¿un laberinto sin salida?
}

JORGE LIENDO TEJADA

Abogado por la Universidad de Lima. Máster en Asesoría Fiscal de Empresas por el Instituto de Empresas - IE Law School.

SUMARI0:

I. Introducción.

II. La cláusula de no-discriminación en la Decisión 578 de la Comunidad Andina.

1. La Decisión 578 como norma comunitaria.

2. El régimen tributario de la Decisión 578.

3. Estructura del artículo 18 de la Decisión 578.

3.1. Países miembros de la Comunidad Andina y la Decisión 578.

3.2. Domicilio en alguno de los Países Miembros de la Comunidad Andina.

3.3. Tratamiento menos favorable.

3.4. Impuestos cubiertos por la Decisión 578.

III. Consideraciones para la aplicación del artículo 18 de la Decisión 578.

IV. Problemática en la aplicación del artículo 18 de la Decisión 578 en los pronunciamientos de la SUNAT: ¿un laberinto sin salida?

1. Primer caso: rentas de trabajo obtenidas en el Perú por un trabajador dependiente domiciliado en otro País Miembro de la Comunidad Andina -Informe No 183-2010-SUNAT/2B0003-.

2. Segundo caso: Informe № 144-2020-SUNAT/7T0000.

2.1. Primer supuesto.

2.2. Segundo supuesto.

2.3. Tercer supuesto.

v. Conclusiones. 


\section{RESUMEN:}

A través de este artículo, el autor desarrolla las problemáticas en la aplicación de la Decisión 578 en los pronunciamientos de SUNAT y llega a la conclusión de que existe una necesidad de explorar y darle mayor contenido a la relación existente entre el ordenamiento jurídico de la Comunidad Andina y la Decisión 578, pues ésta suele revisarse esta de manera aislada y verla como "un convenio más" para evitar la doble imposición.

Palabras clave: Cláusula de no discriminación, Decisión 578, CDI, OCDE, Comunidad Andina, domicilio, TJCAN.

\footnotetext{
ABSTRACT:

In this article, the author develops the problems in the application of Decision 578 in the pronouncements of the Peruvian Tax Administration and concludes that there is a need to explore and give greater content to the relationship between the legal system of the Andean Community and the Decision 578, since the latter is usually studied in isolation and seen as "another treaty" to avoid double taxation.

Keywords: Non-discrimination clause, Decision 578, CDI, OECD, Andean Community, address, TJCAN.
}

\section{INTRODUCCIÓN}

Los diversos modelos de convenios internacionales para evitar la doble imposición' contienen disposiciones que protegen a los "contribuyentes transfronterizos" de tratamientos tributarios discriminatorios en uno de los países contratantes. La doctrina ha denominado a estas disposiciones como "cláusulas de no-discriminación".

En función a políticas fiscales, los sistemas tributarios de cada país establecen tratamientos diferenciados en base a, por ejemplo, la residencia, la nacionalidad, la capacidad contributiva, entre otros. $Y$ es que algunos principios elementales del Derecho Tributario son, entre otros, la equidad vertical y horizontal.

El principio de equidad vertical implica que contribuyentes con diferente capacidad contributiva deben ser tratados de manera diferenciada. Por su parte, bajo el principio de equidad horizontal se establece que contribuyentes bajo condiciones iguales deben ser tratados de igual manera.

Las cláusulas de no-discriminación en los convenios internacionales en materia tributaria tratan de balancear la necesidad de prevenir una situación injustificada de discriminación y la necesidad de tomar en cuenta las distinciones legítimas en la diferenciación de tratamientos tributarios en función a los referidos principios. La Decisión 578 es el régimen para evitar la doble imposición y prevenir la evasión fiscal aprobado por la Comunidad Andina y aplicable a sus países miembros o contratantes; esto es, Bolivia, Colombia, Ecuador y Perú. Esta Decisión tiene en su artículo 18 una prohibición de discriminación basada en la fórmula del domicilio.

El objetivo del presente artículo es volver abrir el debate y el estudio sobre la cláusula de no-discriminación de la Decisión 578 y, por qué no, también sobre el íntegro de esta norma comunitaria, cerca de 17 años desde su expedición.

En tal sentido, abordaremos la composición del artículo 18 de la Decisión 578 para luego analizar su aplicación tomando como referencia los dos únicos pronunciamientos de la Administración Tributaria peruana - SUNAT-, con el propósito de revelar la existencia o no de

1. Los modelos más conocidos y estudiados son los de la Organización para la Cooperación y el Desarrollo Económicos - OECD y el modelo de convenio de las Naciones Unidas. Por ejemplo, el modelo de la OECD incluye cláusulas de no-discriminación para nacionales, personas sin residencia, establecimientos permanentes, para la deducción de gastos y para la tenencia de propiedad sobre ciertos activos. Por cuestiones de especificidad y de espacio, no haremos referencia a las cláusulas de no-discriminación contenidas en el modelo OECD. 
lineamientos interpretativos sobre el referido artículo, contrastando con lo que creemos es la metodología razonable que debería emplearse al aplicar la cláusula de no-discriminación.

Finalmente, resaltaremos nuestras conclusiones incluyendo puntos que consideremos que deben ser analizados y estudiados a mayor profundidad.

\section{LA CLÁUSULA DE NO-DISCRIMINACIÓN EN LA DECISIÓN 578 DE LA COMUNIDAD ANDINA}

\section{La Decisión 578 como norma comunitaria.}

Es importante hacer referencia a un punto que, muchas veces, se suele dejar de lado. La Decisión 578 no es un tratado, es una norma del Derecho Comunitario.

El Derecho Comunitario es la producción normativa, en tanto ordenamiento jurídico, de la voluntad política de diversos Estados de crear un sistema de integración que responde a intereses políticos, económicos y sociales propios de una región y que tiene por principal objetivo, entre otros, facilitar la libertad de movimiento y establecimiento de los ciudadanos de esa región y la libre movilidad de servicios y capitales dentro de la misma.

Como tal, el Derecho Comunitario tiene primacía sobre el derecho nacional para poder garantizar su uniformidad en todos los países miembros. La Unión Europea es, por excelencia, el ejemplo de integración comunitaria de referencia.
En efecto, la supranacionalidad del Derecho Comunitario se manifiesta en dos principios fundamentales: la aplicación directa de la norma comunitaria, que significa que produce efectos jurídicos en los países miembros sin requerir para ello un complemento normativo del derecho interno; $y$, la preeminencia, que es la virtud que tiene el ordenamiento comunitario de primar sobre una norma de Derecho interno que se le oponga. ${ }^{2}$

En el caso de la Comunidad Andina, son dos los principales cuerpos normativos que establecen el ordenamiento jurídico comunitario de la región andina: el Acuerdo de Integración Subregional Andino -en adelante, "Acuerdo de Cartagena" - y el Tratado de Creación del Tribunal de Justicia de la Comunidad Andina - en lo sucesivo, el "Tratado del TJCAN"-.

El Acuerdo de Cartagena crea la Comunidad Andina y establece las normas para su funcionamiento. Los actuales países miembros de la Comunidad Andina son: Bolivia, Colombia, Ecuador y Perú. En adelante, nos referiremos a ellos como los "Países Miembros" o el "País Miembro".

El Tratado del TJCAN señala los órganos e instituciones que conforman el Sistema Andino de Integración; entre ellos, la Comisión de la Comunidad de la Comunidad Andina - en adelante, la "Comisión"- L La Comisión tiene funciones normativas y expresa su voluntad mediante Decisiones, estas forman parte del ordenamiento jurídico de la Comunidad Andina. ${ }^{3}$

En el Derecho Comunitario se distingue entre

2. Luis Carlos Plata López \& Donna Yepes Ceballos, "Naturaleza jurídica de las normas comunitarias andinas", Revista de Derecho 31 (2009): 196-223, http://www.scielo.org.co/scielo.php?script=sci_arttext\&pi$\mathrm{d}=$ S0121-86972009000100008\&lng=en\&tIng=es. Como bien anotan los autores, la primacía del Derecho Comunitario no implica una confusión ni mucho menos la derogación implícita o tácita del Derecho interno de los países miembros; por el contrario, existe una relación de "autonomía recíproca" entre la fuente normativa de la organización supranacional - por ejemplo, la Comunidad Andina-y la de los países miembros. No obstante, en caso de contradicción, se aplica el principio de la primacía del Derecho Comunitario sobre el Derecho interno de los países miembros.

3. Existe otro órgano con atribuciones normativas que también emite Decisiones: el Consejo Andino de Ministros de Relaciones Exteriores. 
normas de Derecho originario o primario y normas de Derecho derivado o secundario. En el caso de la Comunidad Andina, el Acuerdo de Cartagena y el Tratado del TCJCAN son normas de Derecho originario, pues son una suerte de "Constitución" del ordenamiento jurídico comunitario; y, por su lado, la Decisiones son normas de Derecho derivado.

La Comisión adopta sus Decisiones con el voto favorable de la mayoría absoluta de los Países Miembros, y éstas obligan a los Países Miembros desde la fecha en que sean aprobadas por la Comisión. ${ }^{4}$ Es decir, las Decisiones serán directamente aplicables en los Países Miembros a partir de la fecha de su publicación, a menos que las mismas señalen una fecha posterior. ${ }^{5}$

Esto último es importante puesto que es la expresión del principio de aplicación directa, al que hemos hecho referencia.

Entonces, la regla es que la Decisión aplica de manera directa desde su fecha de publicación, y no solo ello, sino que también genera lo que se conoce en doctrina como el "principio efecto directo", según el cual, los ciudadanos de los países miembros de la Comunidad Andina pueden exigir antes las cortes nacionales en cuanto les beneficien y sean aplicables.

La excepción al principio de aplicación directa es la exigencia de transponer la norma comunitaria en el ordenamiento jurídico nacional de cada uno de los Países Miembros. En efecto, el Tratado del TJCAN establece que, cuando su texto así lo disponga, las Decisiones requerirán de incorporación al Derecho interno, mediante acto expreso en el cual se indicará la fecha de su entrada en vigor en cada País Miembro. ${ }^{6}$
En los casos en que así la Decisión lo señale, es necesario que antes de que ésta sea efectivamente aplicable, los Países Miembros la incorporen a su normativa nacional, ya sea mediante la creación de nuevas leyes o la modificación de leyes existentes.

La Decisión 578 establece un régimen para evitar la doble tributación y prevenir la evasión fiscal. Esta Decisión se publicó el 4 de mayo de 2004 y su vigencia empezó el 1 de enero de 2005.

La Decisión 578 no dispuso que deba ser incorporada a la normativa interna de los Países Miembros de la Comunidad Andina, en tal sentido, bajo el principio de aplicación directa, es aplicable y surte plenos efectos para aquellos y sus ciudadanos desde el 1 de enero de 2005.

\section{El régimen tributario de la Decisión 578.}

La Decisión 578 tiene como uno de sus principales objetivos evitar la doble tributación de unas mismas rentas o patrimonios a nivel comunitario que puede surgir cuando una persona o empresa realiza sus actividades económicas en dos o más de los Países Miembros.

Para evitar la doble tributación, como regla general, la Decisión 578 ha recogido la fórmula de la "tributación en la fuente". Esto es, la renta que genere una persona o empresa domiciliada en alguno de los Países Miembros solo podrá estar sujeta a tributación por el País Miembro en donde se haya generado esa renta.

Por ejemplo, si una persona domiciliada en Ecuador realiza un trabajo en Bolivia para una empresa domiciliada en ese país, y este último somete a tributación la contraprestación que

4. Artículo 2 del Tratado del TJCAN.

5. Primer párrafo del Artículo 3 del Tratado del TJCAN.

6. Segundo párrafo del Artículo 3 del Tratado del TJCAN.

7. La Decisión 578 sustituyó a la Decisión 40 que estableció un régimen distinto para evitar la doble tributación. 
aquella pague por el trabajo realizado, solo tributaría en Bolivia y no en Ecuador.

Salvo Bolivia, el resto de los Países Miembros someten a tributación a personas y empresas domiciliadas por sus rentas locales mundiales; y a aquellos que no son domiciliados solo por sus rentas locales. ${ }^{8}$ Así, si un peruano con domicilio en Perú realiza trabajo en ese país, pero obtiene dividendos en Ecuador, en ausencia de la Decisión 578, debería tributar en Perú por el trabajo realizado y por los dividendos percibidos y sobre estos últimos también tendría que tributar en Ecuador. Esta última situación es evitada por la Decisión, al disponerse, siguiendo el criterio de tributación en la fuente, que los dividendos solo tributan en Ecuador y no en Perú -este último deberá reconocer tales dividendos como exonerados del Impuesto a la Renta-.

La ecuación es sencilla: primero debemos revisar las normas tributarias internas de cada País Miembro para saber si una determinada renta está gravada o no. Si la renta se encuentra gravada en más de un País miembro, entonces debemos recurrir a la Decisión 578 y determinar el lugar de fuente de dicha renta. La renta tributará en el País Miembro en donde se encuentre la fuente y el otro País Miembro deberá considerar la misma renta como exonerada. El siguiente paso es revisar la cláusula de no-discriminación.

\section{Estructura del artículo 18 de la Decisión 578.}

La cláusula de no-discriminación de la Decisión 578 la encontramos en su artículo 18 que establece lo siguiente:

"Ningún País Miembro aplicará a las personas domiciliadas en los otros Países Miembros, un tratamiento menos favorable que el que aplica a las personas domiciliadas en su territorio, respecto de los impuestos que son materia de la presente Decisión".

Para analizar esta regla, podemos descomponer su lenguaje o redacción de la siguiente manera:

- $\quad$ ningún país miembro;

- aplicará a las personas domiciliadas en los otros países miembros;

- $\quad$ un tratamiento menos favorable;

- $\quad$ que el que aplique a las personas domiciliadas en su territorio; $y$,

- respecto de impuestos que son materia de la presente Decisión.

\subsection{Países miembros de la Comunidad Andi- na y la Decisión 578.}

Hemos mencionado que los actuales Países Miembros de la Comunidad Andina son Bolivia, Colombia, Ecuador y Perú.

Sobre este punto vale la pena hacer una acotación. Adolfo Martín señala que el principio de no-discriminación en los Convenios para Evitar la Doble Imposición no opera al margen de tales convenios; es decir, no existe un principio general de no-discriminación en Derecho Tributario internacional. ${ }^{9}$ Esto es cierto en la medida en que dichos convenios se suscriben de manera bilateral entre dos países y es el instrumento que regula las relaciones tributarias entre ambos.

No obstante, ello no es de todo cierto en el caso que analizamos. Hemos precisado en detalle que la Decisión 578 no es un tratado internacional, sino es parte de un ordenamiento jurídico supranacional, dentro del cual existen principios generales que buscan asegurar la misión de la Comunidad Andina. En tal sentido, la cláusula de no-discriminación de la Decisión 578 no solo se circunscribe al resto de sus disposicio-

8. En Bolivia aplica un sistema de tributación territorial; donde, tanto para domiciliados como para no domiciliados, solo se gravan las rentas que tengan su origen en Bolivia.

9. Adolfo Martín Jiménez, “El principio de no discriminación en el artículo 24 del modelo de convenio de la OCDE de 2008", Revista de Derecho Fiscal 5 (1) (2011), https://revistas.uexternado.edu.co/index.php/fiscal/article/view/2751. 
nes, sino también a las normas comunitarias y a la jurisprudencia del TJCAN.

\subsection{Domicilio en alguno de los Países Miem- bros de la Comunidad Andina.}

Para estar ante un supuesto de aplicación del citado artículo 18 de la Decisión 578, es necesario que hablemos de personas domiciliadas en los Países Miembros de la Comunidad Andina y no de un tercer país.

En otras palabras, podemos ver que la cláusula de no-discriminación de la Decisión 578 ha tomado como elemento de referencia el domicilio fiscal de las personas. Esta cláusula proscribe la discriminación en función al domicilio fiscal de las personas domiciliadas en algunos de los Países Miembros de la Comunidad Andina.

El domicilio de las personas viene, en primer lugar, determinado por la norma interna de cada País Miembro. Así, por ejemplo, en el caso de Perú, se considera que, para efectos tributarios, las personas naturales de nacionalidad peruana son domiciliadas siempre que tengan domicilio en el país de acuerdo con las reglas de derecho común. ${ }^{10}$

Por otro lado, una persona natural extranjera es domiciliada en el país cuando haya residido o permanecido en Perú más de 183 días calendario dentro de un período cualquiera de 12 meses."

Las reglas de domicilio fiscal en Perú son relativamente sencillas. Esto no es así en todos los países. Por ejemplo, Colombia establece reglas de domicilio fiscal para nacionales colombianos que tengan el $50 \%$ o más de sus activos poseídos en Colombia.

Así, podríamos tener un caso en el que un nacional colombiano permanece en Perú por más de 183 días, pero tiene todos sus bienes y pro- piedades en Colombia. Estamos ante un caso de doble domicilio fiscal.

Este conflicto de domicilio fiscal es resuelto por la misma Decisión 578 en su artículo 2(e), mediante la siguiente regla: una persona física o natural será considerada domiciliada en el País Miembro en que tenga su residencia habitual.

Si el criterio de "residencia habitual" no es suficiente para resolver el conflicto de domicilio fiscal, las autoridades tributarias de los Países Miembros deberán resolver el caso de común acuerdo.

\subsection{Tratamiento menos favorable.}

Específicamente, la cláusula de no-discriminación proscribe un "tratamiento menos favorable" que el que aplique a las personas domiciliadas en un País Miembro cuando en éste se genere una renta en cabeza de una persona domiciliada en otro País Miembro.

Dicho de otra forma, dicha cláusula cubre la discriminación impuesta por y manifestada en uno de los Países Miembros con respecto a una persona domiciliada en otro País Miembro.

Es importante destacar que la frase "tratamiento menos favorable" es evidencia de que el artículo 18 ha sido redactado en una terminología de no discriminación, en oposición a una terminología igualitaria o equitativa. Nótese que la regla no obliga a los Países Miembros a tratar a una persona domiciliada en otro País Miembro de manera igual a la que trata a sus domiciliados. El artículo 18 de la Decisión 578 prohíbe un tratamiento menos favorable y no uno más favorable en perjuicio de las personas domiciliadas en el País Miembro que se analice.

Si bien es cierto que esta distinción parece ser solo teórica, sí es relevante, pues nos informa sobre el alcance del artículo 18.

\footnotetext{
10. Artículo 7, inciso a) del Decreto Supremo N 179-2004-EF, Texto Único Ordenado de la Ley del Impuesto a la Renta -LIR.

11. Articulo 7, inciso b) de la LIR.
} 
En la misma línea, Tulio Tartarini explica que del artículo 18 de la Decisión 578 no emana un tratamiento equitativo entre personas domiciliadas en la Comunidad Andina. Señala que sostener esta postura nos llevaría a pensar que una persona domiciliada en uno de los Países Miembros debe ser sometida a imposición bajo los mismos términos en todos los otros Países Miembros. El efecto sería que los domiciliados en los Países Miembros de la Comunidad Andina sean tratados como domiciliados en todos esos países. ${ }^{12}$

Coincidimos con la opinión de que este no es el caso y que lo que persigue el artículo 18, siguiendo al mismo autor, es que las rentas estén sometidas a imposición en una sola jurisdicción y de la forma en que dicha jurisdicción las grava para sus propios domiciliados.

Cada País Miembro sigue siendo libre de determinar los supuestos que generan una renta gravada bajo su jurisdicción; los cuales, naturalmente, deben luego contrastarse con la Decisión 578.

Por otro lado, la Decisión 578 no define qué debe entenderse por "menos favorable"; en ese sentido, a falta de indicación expresa en contrario y en base a prácticas internacionales, el tratamiento menos favorable no solo debe constituir el pago o determinación de un mayor impuesto; sino también, la imposición de mayores cargas formales.

\subsection{Impuestos cubiertos por la Decisión 578.}

Finalmente, la cláusula de discriminación de la Decisión solo aplicará respecto de los impuestos cubiertos por la misma; esto es, según su artículo 1, a los impuestos sobre la renta y sobre el patrimonio.
En el caso de Perú, esta disposición alcanza a aquellas obligaciones tributarias que se deriven de la aplicación del Impuesto a la Renta. No existe en nuestro país un impuesto al patrimonio.

Así, por ejemplo, la Decisión y su cláusula de no-discriminación no alcanza a impuestos como el Impuesto General a las Ventas, Impuesto de Alcabala, entre otros.

\section{CONSIDERACIONES PARA LA APLICACIÓN DEL ARTÍCULO 18 DE LA DECISIÓN 578}

Es ampliamente aceptado que una situación de discriminación solo puede existir cuando todos los factores a tomar en cuenta son similares y el trato diferenciado - o trato menos favorable en los términos de la Decisión 578- se basa exclusivamente en la diferencia que es prohibida por la norma en cuestión; en nuestro caso, el artículo 18 de la Decisión 578 que incluye al domicilio como "diferencia prohibida".

Siguiendo al académico Niels Bammens ${ }^{13}$, que nos presenta una aproximación aristotélica de una cláusula de no-discriminación, cuatro elementos se pueden distinguir en la concepción de discriminación: (i) dos situaciones (ii) son tratadas de manera diferente (iii) a pesar de ser comparables, (iv) resultando en una desventaja para una de esas situaciones.

Conceptualmente, es importante llamar la atención sobre el primero de los elementos mencionados, puesto que para poder hablar de dos situaciones al menos una característica de estas debe ser diferente; de lo contrario no sería posible hablar de discriminación. Es por ello también que hablamos de situaciones comparables y no de situaciones idénticas o iguales.

12. Tulio Tartarini Tamburini, “El principio de no discriminación contenido en la Decisión 578 de la Comunidad Andina de Naciones y las rentas de capital", THEMIS Revista De Derecho 64 (2013): 181-195. http://revistas.pucp.edu.pe/ index.php/themis/article/view/9581.

13. Niels Bammens, "The principle of non-discrimination in international and European tax law", IBFD Doctoral Series Volumen 24 (2012): 9, https://books.google.com.pe/books?id=b3LGM41RpdMC\&pg=PA9\&source=gbs_toc_r\&$\mathrm{cad}=4 \# \mathrm{v}=$ onepage $\& \mathrm{q} \& \mathrm{f}=\mathrm{false}$. 
Para la aplicación de una cláusula de discriminación, siguiendo doctrina y jurisprudencia europea, se requiere de dos tests: (i) test de comparabilidad; $y$, (ii) y test de desventaja ${ }^{14}$ :

(i) Test de comparabilidad: una cláusula de no-discriminación solo aplica si hay dos sujetos que son comparables entre sí, en situaciones comparables.

(ii) Test de desventaja: si las situaciones son comparables, uno de los elementos de comparación conlleva a un tratamiento diferente que es menos favorable para uno de los sujetos comparados, únicamente por disposición expresa de una norma específica que toma en cuenta dicho elemento - en nuestro caso, el domicilio-.

Estando a ello, a continuación analizaremos los dos únicos casos en los que la SUNAT se ha pronunciado sobre la aplicación del artículo 18 de la Decisión 578.

\section{PROBLEMÁTICA EN LA APLICACIÓN DEL ARTÍCULO 18 DE LA DECISIÓN 578 EN LOS PRONUNCIAMIENTOS DE LA SUNAT: ¿UN LABERINTO SIN SALIDA?}

\section{Primer caso: rentas de trabajo obteni- das en el Perú por un trabajador depen- diente domiciliado en otro País Miembro de la Comunidad Andina - Informe No 183-2010-SUNAT/2B0003-.}

La pregunta planteada a SUNAT fue qué tratamiento tributario aplica en nuestro país a la renta obtenida por la persona indicada. Para efectos prácticos, supongamos que se trata de una persona nacional y domiciliada en Ecuador.

Para empezar, recodemos que, de acuerdo con la LIR, se consideran rentas de fuente pe- ruana las obtenidas por el trabajo personal, en el ejercicio de una profesión.

Sobre este tipo de rentas, las personas domiciliadas en el país tributan con una escala acumulativa progresiva que va desde el $8 \%$ al $30 \%$, previa deducción de algunos conceptos establecidos por la referida Ley, como las conocidas 7 Unidades Impositivas Tributarias - UIT- de "supervivencia".

En cambio, tratándose de personas naturales no domiciliadas en el Perú que obtengan la misma renta de trabajo, tributan con una tasa del $30 \%$ sobre el monto bruto de la remuneración que perciban por el trabajo realizado en el país, sin la posibilidad de aplicar deducción alguna.

En el presente caso tenemos como sujeto comparable a la persona domiciliada en Ecuador y como objeto comparable a la persona domiciliada en el Perú —es esta última contra quien hacemos la comparación de la persona domiciliada en Ecuador-. Siguiendo la línea de análisis que proponemos, veamos como resuelve SUNAT.

a) Sujeto comparable: trabajador dependiente domiciliado en Ecuador.

b) Objeto comprable: trabajador dependiente domiciliado en Perú.

c) Renta de fuente peruana sujeta a tributación: rentas de trabajo generadas en relación de dependencia.

d) Aplicación de la Decisión 578: la SUNAT determina si resulta aplicable la Decisión 578 en función al domicilio de la persona no domiciliada que genera renta de trabajo en el Perú. Concluye que sí, puesto que en el caso materia de consulta, la persona do-

14. Niels Bammens y Frans Vanistendael, Global Tax treaty Commentaries - Article 24: Non-Discrimination, (IBFD, 2016), citado en Francesco De Lillo, "Chapter 19: Prohibition of Discrimination", en Roy Rohatgi on International Taxation - Volume 1: Principles, eds. Ola Ostaszewska y Belema Obuoforibo (Amsterdam: IBFD, 2018): 357. 
miciliada en Ecuador no había pasado más de 183 días en Perú.

Seguidamente, el Informe analiza el artículo 13 de la Decisión 578 para determinar qué País Miembro tiene la potestad para someter a tributación la renta de trabajo generada. Como vimos, la Decisión 578 consagra el criterio de la fuente. En este caso, la persona de nacionalidad ecuatoriana realizaba su trabajo en Perú por lo tanto, es Perú quien grava y Ecuador debe reconocer la misma renta como exonerada.

e) Aplicación del artículo 18 de la Decisión 578: la SUNAT señala que el artículo 18 "especificamente prohíbe el trato discriminatorio en función al domicilio del sujeto, razón por la cual, en el supuesto bajo análisis, una persona natural domiciliada en un país miembro de la Comunidad Andina que esté sujeta al Impuesto a la Renta en el Perú tiene derecho a tributar, desde el primer día de su permanencia en el país, de modo equivalente a como tributan las personas naturales domiciliadas en el Perú, salvo que existiera un tratamiento diferenciado en función a alguna consideración adicional distinta al domicilio" - el subrayado es nuestro-.

Seguidamente, citando a Fernando Zuzunaga, la SUNAT precisa que "para que proceda la aplicación de la Cláusula de No Discriminación de la Decisión 578 es necesario que se presenten los requisitos o características que la ley interna del país exige a sus domiciliados para la aplicación de dicha ley excepto el ser domiciliado".15

Comentario: nótese que la SUNAT correctamente indica que, si el único elemento diferenciador del tratamiento tributario a la persona domiciliada en Ecuador es el domicilio, entonces ésta deberá tributar en el Perú como tributa una persona domiciliada en este país.
Si la LIR hubiese incorporado otro elemento diferenciador distinto al domicilio, el tratamiento menos favorable o la desventaja para el nacional ecuatoriano estaría permitida.

Ahora bien, la cita de Fernando Zuzunaga empleada por la SUNAT no es desarrollada y el autor no brinda mayor detalle, aunque sí una pista. Esa cita, según el autor, atiende a los fundamentos del principio de igualdad horizontal.

Creemos que lo que se ha tratado decir en este punto es que para aplicar el test de comparabilidad y desventaja que hemos mencionado, debemos partir de la ley interna del país, en este caso de Perú. Veamos.

(i) Test de comparabilidad: los sujetos comparables en este caso son ambos personas naturales; ambos realizan un trabajo en el Perú; ambos realizan su trabajo de manera dependiente; $y$, ambos reciben una remuneración por el trabajo realizado, manifestando cierto grado de capacidad contributiva. La LIR grava las contraprestaciones que perciban sus domiciliados por trabajos dependientes llevados a cabo en territorio nacional; esto también es así para personas no domiciliadas. La similitud es evidente. Se cumple con el test de comparabilidad.

(ii) Test de desventaja: por más que pueda resultar sencillo, es importante seguir el hilo lógico de aplicación de la cláusula de no-discriminación, pues podría haber casos más complejos, como veremos más adelante.

En el presente caso, como ya hemos adelantado, el factor domicilio hace que el nacional ecuatoriano esté sujeto a una mayor carga impositiva que el domiciliado peruano que, en circunstancias comparables,

15. Fernando Zuzunaga del Pino, "Tema 2: Convenios para evitar la doble imposición" (ponencia general, IX Jornadas Nacionales de Tributación IFA, 4 de abril de 2008). 
soporta una menor carga ya que a este último se le permite tomar deducciones y aplicar una tasa del impuesto marginal. Se verifica el test de desventaja.

Estando a ello, con seguridad podemos afirmar que resulta de aplicación el artículo 18 de la Decisión 578.

f) Conclusión de SUNAT: “(...) teniendo en cuenta lo dispuesto en el artículo 18 de la Decisión 578, dichas personas (las domiciliadas en otros Países Miembros), aun cuando no califiquen como domiciliadas en el país (Perú), tendrán derecho a determinar el Impuesto a la Renta considerando la deducción anual de siente Unidades Impositivas Tributarias establecidas en el artículo 46 del TUO de la Ley del Impuesto a la Renta y la escala progresiva acumulativa prevista en el artículo 53 del mismo TUO, toda vez que la legislación nacional del Impuesto a la Renta ha previsto diferencias para el cálculo de la obligación tributaria en función a la existencia o no de la condición de domiciliado en el Perú" —el subrayado es nuestro-.

Comentario: la corrección de la desventaja generada por el factor domicilio es reconocer en favor del nacional ecuatoriano la deducción de las 7 UIT y la aplicación de la escala acumulativa progresiva para la determinación de su Impuesto a la Renta peruano, tal y como si se considerase domiciliado en el Perú.

Consideramos que la conclusión a la que llega la SUNAT es jurídicamente correcta. El criterio más importante que nos deja es que para el test de comparabilidad y desventaja debemos tomar como punto de partida la norma interna del País Miembro que está sujetando a tributación la renta en cuestión.

Ahora bien, y resulta curioso, la SUNAT no adopta todas la observaciones de Fernando Zuzunaga quien señala, poniéndose en el supuesto de que el trabajador nacional ecuatoriano solo trabaja 5 meses en el Perú, que dada la configuración del Impuesto a la Renta peruano de sujetos domiciliados, la deducción de las 7 UIT es anual y la escala progresiva acumulativa es aplicable sobre la renta anual y de fuente mundial, no existe un mecanismo previsto para aplicar la cláusula de no-discriminación en caso de rentas obtenidas por un no domiciliado en cinco meses, razón por la cual en dicho caso la referida cláusula no será efectiva.

Aunque es razonable, no compartimos esta opinión; toda vez que, si tuviéramos una persona domiciliada en el Perú que solo trabaja 5 meses y no tiene otra fuente de ingreso, ni local ni extranjera, igual tendrá derecho a la deducción de las 7 UIT, estará sujeto a la escala acumulativa progresiva y deberá presentar su declaración jurada anual del Impuesto a la Renta, de corresponder. Es decir, la forma de cálculo seguirá siendo la misma.

No obstante, si reconocemos que existen dificultades de orden administrativo para una implementación correcta del artículo 18 de la Decisión 578. La SUNAT podría establecer formularios virtuales y especiales para estos casos, así como, lineamientos informativos.

\section{Segundo caso: Informe No 144-2020-SUNAT/7T0000.}

Los tres supuestos planteados en este informe son complejos. No negamos la dificultad que enfrenta la SUNAT para resolver estos supuestos; sin embargo, buscando una solución simplista, la Administración Tributaria deja pasar una oportunidad para brindar luces sobre la aplicación e interpretación del artículo 18 de la Decisión 578. Una vez más, la SUNAT deja pasar la oportunidad de contribuir con la seguridad jurídica y de generar confianza en los contribuyentes.

\subsection{Primer supuesto.}

Rendimientos obtenidos por sociedad Administradora de Fondos de Pensiones -AFP colombiana como resultados de sus inversiones en el Perú, efectuadas a través de fondos de inversión o fideicomisos constituidos en Colombia.

El motivo de la consulta se debe a que tratán- 
dose de AFP domiciliadas en el Perú, y que como resultado de sus inversiones en el país, efectuadas a través de fondos de inversión o fideicomisos constituidos en el Perú, obtienen rendimientos que no se encuentran gravados con el Impuesto a la Renta en Perú: ¿debe otorgarse el mismo tratamiento para AFP y fondos de inversión o fideicomisos constituidos en Colombia que obtienen rentas en Perú?

Resulta un poco complicado seguir la ilación de la lógica empleada por la SUNAT, pero para una mayor claridad, sigamos con nuestro método propuesto:

a) Sujeto comparable: fondos de inversión o fideicomisos domiciliados en Colombia

Comentario: como bien apunta la SUNAT, el sujeto que obtiene rentas de fuente peruana en el supuesto de la consulta es el fondo de inversión o fideicomiso constituido en Colombia, no las AFP.

b) Objeto comparable: fondos de inversión o fideicomisos domiciliados en Perú.

c) Renta de fuente peruana sujeta a tributación: en este extremo hay silencio de la SUNAT, quien se limita a señalar que "siendo que en el supuesto consultado no se precisa el tipo de rentas que obtiene el fondo de inversión o fideicomiso constituido en Colombia, por lo que dicha situación deberá evaluarse en cada caso en particular" -el subrayado es nuestro-.

Comentario: esta posición de SUNAT nos imposibilita a continuar con nuestro análisis pues si no conocemos de qué renta hablamos ¿cómo determinamos si se sujeta a tributación en el Perú o no?

Sin perjuicio de ello, la SUNAT pudo muy bien haber asumido las rentas que generan los fondos de inversión o fideicomisos. Principalmente, en base a la naturaleza de estos vehículos, las rentas que estos generan son rentas pasivas: intereses, dividendos y ganancias de capital.
Como bien indica la SUNAT, el artículo 14 de la LIR establece que son contribuyentes de dicho impuesto entre otros, las personas jurídicas, siendo que el inciso f) precisa que se consideran personas jurídicas a las empresas unipersonales, las sociedades y las entidades de cualquier naturaleza, constituidas en el exterior, que en cualquier forma perciban renta de fuente peruana.

Bajo el derecho común, los fondos de inversión y fideicomisos se caracterizan por ser patrimonios autónomos, es decir, entidades sin personería jurídica; no obstante, para efectos tributarios, este tipo de entidades se consideran como personas jurídicas -empresas-.

Por lo tanto, los fondos de inversión y fideicomisos extranjeros estarán sujetos al Impuesto a la Renta por sus rentas de fuente peruana. La consulta sí es clara en el sentido de que las inversiones de estos fondos se realizan en Perú.

Si bien la consulta no especifica la renta pasiva exacta, ésta tiene su origen en Perú $y$, como bien precisa la SUNAT, adelantándose al análisis de la potestad tributaria en la Decisión 578, a pie de página: "con el artículo 10 los intereses y demás rendimientos financieros sólo serán gravables en el País Miembro en cuyo territorio se impute y registre su pago; mientras que conforme al artículo 11 los dividendos y participaciones sólo serán gravables por el País Miembro donde estuviera domiciliada la empresa que los distribuye. Por su parte, el artículo 12 establece que las ganancias de capital sólo podrán gravarse en el País miembro en cuyo territorio estuvieran situados los bienes al momento de su venta. Con excepción de las ganancias de capital obtenidas por la enajenación de: (i) naves, aeronaves, autobuses y otros vehículos de transporte, que sólo serán gravables por el País Miembro donde esta domiciliado el propietario, y (ii) títulos, acciones, otros valores, que sólo serán gravables por el País Miembro en cuyo territorio se hubieran

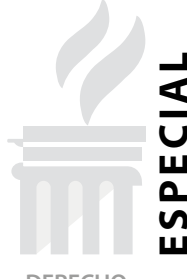


emitido". Si, como veremos, la SUNAT agota su análisis ¿por qué hacer referencia a estos artículos de la Decisión 578?

Consideramos que con estas precisiones la SUNAT pudo continuar con el análisis del presente caso.

d) Aplicación de la Decisión 578: en sus conclusiones la SUNAT señala que "para efectos de la aplicación de la cláusula de no discriminación se debe establecer en cada caso en particular si el fondo de inversión o fideicomiso califica como "empresa" domiciliada en Colombia, así como el tratamiento tributario de acuerdo al tipo de renta que se obtenga".

Comentario: nuevamente la SUNAT nos lleva a un callejón sin salida.

Si superamos el hecho de que no se especifica la renta en particular del presente caso y que esta se encuentra gravada en Perú, el paso siguiente es determinar si resulta aplicable la Decisión 578; y primero debemos revisar si los fondos de inversión o fideicomisos colombianos son residentes en Colombia para efectos de dicha Decisión.

El artículo 1 de la Decisión 578 establece que esta es aplicable a las personas domiciliadas en cualquiera de los Países Miembros de la Comunidad Andina.

El literal c) del artículo 2 de la Decisión 578 prevé que el término "persona" servirá para designar a:

(i) Una persona física o natural.

(ii) Una persona moral o jurídica.

(iii) Cualquier otra entidad o grupo de personas, asociadas o no, sujetas a responsabilidad tributaria.

Hemos mencionado que los fondos de inversión y fideicomisos tienen una categoría especial. Es necesario conocer cuál su tratamiento en la normativa interna colombiana para saber si califican como persona jurídica, en cuyo caso sí aplicaría la Decisión; o, si de lo contrario, son entidades sujetas al pago de impuestos en Colombia, siendo que, de igual manera, resultaría de aplicación la Decisión.

La SUNAT se limita a mencionar que este análisis de domicilio se debe hacer caso por caso y así evita pronunciarse sobre el fondo de la consulta.

Consideramos que, en aras del cumplimiento de los fines del sistema comunitario andino, particularmente de la libertad de movimiento de capitales, la SUNAT pudo confirmar con la autoridad tributaria colombiana el tratamiento que reciben los fondos de inversión y fideicomisos en Colombia para confirmar si son domiciliados según la Decisión 578; con una simple carta hubiese bastado. Ahora, si esto representaba una dificultad insuperable para la SUNAT, bien pudo asumir que los fondos de inversión y fideicomiso colombianos sí son domiciliados bajo la Decisión 578 y continuar con el análisis.

e) Aplicación del artículo 18 de la Decisión 578: sin perjuicio de lo anterior la SUNAT establece que "en caso se cumpla con la premisa de que el fondo de inversión o fideicomiso califica como empresa domiciliada en Colombia de acuerdo con los términos de la Decisión 578, a fin de evaluar la aplicación de la cláusula de no discriminación se debe comparar el tratamiento tributario que recibe la empresa domiciliada en Colombia con el otorgado a una persona jurídica domiciliada en el Perú por el mismo tipo de renta, siendo que en el supuesto consultado no se precisa el tipo de rentas que obtiene el fondo de inversión o fideicomiso constituido en Colombia, por lo que dicha situación deberá evaluarse en cada caso en particular" - el subrayado es nuestro-.

Comentario: por un momento la SUNAT asume que los fondos de inversión y fideicomiso colombianos sí son domiciliados bajo la Decisión 578 pero decide no continuar con el análisis porque la consulta no 
especifica la renta que estas entidades obtienen en el Perú. Otro callejón sin salida. Seguimos sin escapar del laberinto.

Con ello, la SUNAT se escapa de aplicar los tests de comparabilidad y de desventaja.

f) Conclusión de SUNAT: "Ios rendimientos obtenidos por inversiones en el Perú de fondos de inversión o fideicomisos domiciliados en Colombia se encuentran gravados con el Impuesto a la Renta en el Perú, siempre que correspondan a supuestos gravados en la LIR, calificando el referido fondo de inversión o fideicomiso como contribuyente del impuesto en calidad de persona jurídica no domiciliada, resultando irrelevante que dichas rentas sean atribuidas a terceros bajo la legislación colombiana como sería el caso de una AFP; siendo que para efectos de la aplicación de la cláusula de no discriminación se debe establecer en cada caso en particular si el fondo de inversión o fideicomiso califica como "empresa" domiciliada en Colombia, así como el tratamiento tributario de acuerdo al tipo de renta que se obtenga" -el subrayado es nuestro-.

Comentario: esto es simplemente el resumen de los puntos antes mencionados. La SUNAT no brinda una respuesta a la consulta.

\subsection{Segundo supuesto.}

Rendimientos obtenidos por los activos que respaldan las reservas técnicas de las compañías de seguros de vida constituidas o establecidas en Colombia, como resultado de sus inversiones en el Perú, efectuadas a través de fondos de inversión o fideicomisos constituidos en Colombia.

Al igual que el caso supuesto anterior, la SUNAT se limita a señalar que para que aplique la De- cisión deberá estudiarse cada caso en particular. En tal sentido, para este segundo supuesto, nuestros comentarios son los mismos a los arriba expuestos.

\subsection{Tercer supuesto.}

Fundaciones legalmente establecidas en Colombia cuyo instrumento de constitución comprenda exclusivamente alguno o varios de los fines contemplados en la LIR que permiten considerar a fundaciones domiciliadas como entidades exoneradas de dicho impuesto.

a) Sujeto comparable: fundaciones domiciliadas en Colombia que tienen los mismos fines sociales que otorgan la exoneración tributaria en Perú a este tipo de entidades.

b) Objeto comparable: fundaciones domiciliadas en Perú exoneradas del Impuesto a la Renta por cumplir con los fines sociales reconocidos por la LIR.

Comentario: para recordar, la LIR establece una inafectación de dicho impuesto para las fundaciones legalmente establecidas que se dediquen exclusivamente a alguno o varios de los fines señalados en el inciso c) del artículo 18 de dicha Ley. ${ }^{16}$

c) Renta de fuente peruana sujeta a tributación.

Comentario: no hay referencia alguna en el informe sobre el tipo de renta que generan las Fundaciones.

d) Aplicación de la Decisión 578.

Comentario: la SUNAT no hace un examen de domicilio como si lo hace para los anteriores supuestos. Por el contrario, deja entrever que considera a las fundaciones legalmente establecidas en Colombia como domiciliadas para efecto de la Decisión 578.

16. Los fines previstos por la norma son los siguientes: cultura, investigación superior, beneficencia, asistencia social y hospitalaria y beneficios sociales para los servidores de las empresas. 
e) Aplicación del artículo 18 de la Decisión 578: como premisa, la SUNAT señala, citando nuevamente a Fernando Zuzunaga, que "dado que la exigencia de no discriminación es aplicable únicamente al factor domicilio fiscal, es posible otorgar un tratamiento diferenciado en función a otros factores, como por ejemplo los fines de la sociedad, calificación de persona jurídica y persona física, etc. Así, si la legislación de un País Miembro otorga a un domiciliado en otro País Miembro un tratamiento diferenciado que se sustenta no sólo en la diferente condición de domicilio sino en algún otro factor, no aplica la Cláusula de No Discriminación" - el subrayado es nuestro-.

Continúa señalando que "(..) no se transgrede el principio de no discriminación contenido en la Decisión 578, cuando el País Miembro que resulte competente para gravar con el impuesto sobre la renta a un sujeto no domiciliado en ese país, no aplique a dicho sujeto la legislación que le corresponde a sus domiciliados, debido a factores diferentes al domicilio".

Entrando al supuesto planteado, la SUNAT hace referencia a que, en base al inciso f) del artículo 14 de la LIR al que hemos hecho mención antes, "las fundaciones legalmente establecidas en Colombia que perciben rentas de fuente peruana son consideradas contribuyentes del Impuesto a la Renta en calidad de personas jurídicas no domiciliadas $y$, por ende, sujetos pasivos del referido impuesto".

Para la aplicación del artículo 18 de la Decisión 578, la SUNAT señala que "(...) debe verificarse si existe un trato discriminatorio atendiendo a la calidad de persona jurídica no domiciliada de la fundación legalmente establecida en Colombia (sujeto que percibe las rentas), en comparación con el tratamiento que sería aplicable a una persona jurídica domiciliada en el Perú, respecto del mismo tipo de renta y operaciones, careciendo de objeto analizar los fines establecidos en el instrumento de constitución de la funda- ción establecida en Colombia, pues el factor a evaluar es la discriminación en función del domicilio del sujeto" -el subrayado es nuestro-.

Comentario: Traduzcamos el análisis de SUNAT en base a los tests de comparabilidad y desventaja:

(i) Test de comparabilidad: tanto las fundaciones colombianas como las peruanas tienen el mismo propósito; esto es, son entidades sin fines de lucro y persiguen alguno de los fines señalados en la Ley del Impuesto a la Renta. Ambas son consideras para efectos tributarios como personas jurídicas. Ambas se encuentran sujetas a tributación en Perú por sus rentas de fuente peruana. Consideramos que el grado de comparabilidad es razonable y que, por lo tanto, se cumple este test.

(ii) Test de desventaja: las fundaciones que cumplan con los requisitos - fines- se encuentran infectas del Impuesto a la Renta; sin embargo, la norma no deja en claro si dicha inafectación —no sujeción - es solo aplicable a fundaciones domiciliadas.

El inciso c) del artículo 18 de la LIR señala que, no son sujetos pasivos del impuesto (...) las "fundaciones legalmente establecidas, cuyo instrumento de constitución comprenda exclusivamente alguno o varios de los siguientes fines (...); fines cuyo cumplimiento deberá acreditarse con arreglo a los dispositivos legales vigentes sobre la materia" - el subrayado es nuestro-.

La norma menciona "fundaciones legalmente establecidas". No dice establecidas en Perú. Tampoco dice establecidas en cualquier jurisdicción.

Parece que hemos llegado a un punto en nuestro laberinto en el que no sabemos qué dirección tomar. 
Por un lado, siguiendo el criterio de SUNAT de que carece de objeto analizar los fines del referido artículo, solo tendríamos dos resultados posibles pero que nos llevan a la misma conclusión. Primero, si el inciso c) del artículo 18 de la LIR se refiere a fundaciones domiciliadas y no domiciliadas - no distinguiendo donde la ley no lo hace-, entonces no habría desventaja alguna, puesto que las fundaciones legalmente establecidas en Colombia y en Perú, ambas estarían inafectas del Impuesto a la Renta. Esta no pareciera ser la interpretación de SUNAT. Segundo, el inciso c) del artículo 18 de la LIR solo se refiere a fundaciones legalmente establecidas y domiciliadas en el Perú, en cuyo caso sí existiría una desventaja basada en el domicilio, pues por ello las fundaciones colombianas no pueden acceder a la inafectación, en cuyo caso esto debe ser corregido por el artículo 18 de la Decisión 578 y concluir que éstas también se encuentran inafectas del Impuesto a la Renta. No creemos que esta sea la conclusión de la SUNAT.

Por otro lado, podría tomarse la posición de que el inciso c) del artículo 18 de la Ley del Impuesto a la Renta solo se refiere a fundaciones legalmente establecidas y domiciliadas en Perú. En este caso, ¿se verificaría el test de desventaja por que en base al domicilio la fundación colombiana no accedería a la inafectación? La respuesta tendría que ser que no. En efecto existe una desventaja, pero esta se concibe en base a los fines a que se refiere dicha disposición y no al domicilio; entonces, los fines sí importan, no tanto por su contenido sino como factor o presupuesto para la aplicación del inciso c) del artículo referido. No es claro, pero creemos que esta es la posición de la SUNAT.

f) Conclusión de la SUNAT: “(...) las fundaciones legalmente establecidas en Colombia que perciben rentas de fuente peruana, son consideradas contribuyentes del Impuesto a la Renta en el Perú y sujetos pasivos de dicho impuesto, por lo que el impuesto que se aplica sobre estos sujetos no debe ser más gravoso que aquel que se aplica a una persona jurídica domiciliada en el Perú por el mismo tipo de renta, de acuerdo a lo dispuesto en el artículo 18 de la Decisión 578, lo cual debe evaluarse en cada caso en particular" -el subrayado es nuestro-.

Comentario: la SUNAT no se arriesga a ser categórica pero se sigue de su conclusión que, en su opinión, no existe discriminación bajo el artículo 18 de la Decisión 578 al comparar una fundación colombiana y una fundación peruana exonerada del Impuesto a la Renta pese a existir una clara desventaja para la primera, toda vez que esta no se debe a un criterio de domicilio sino al objeto o fines de la fundación, supuesto que no ha sido recogido por la Decisión 578 en tanto es distinto al factor domicilio.

Lo que si señala la SUNAT es que el gravamen que debe soportar las fundaciones no debe ser menos favorable que el que soportan las personas jurídicas -empresas- en Perú. Debiendo referirse al gravamen bajo el régimen general de rentas de tercera categoría; es decir, las fundaciones colombianas, bajo el artículo 18 de la Decisión 578, tienen derecho a tributar sobre renta neta - deduciendo costos y gastos- a una tasa del $29.5 \%$, en lugar de una tasa del $30 \%$ sobre renta bruta.

\section{v. CONCLUSIONES}

a) A 17 años de su publicación, el artículo 18 de la Decisión 578 sigue siendo un laberinto sin una salida clara y que brinde seguridad tributaria a los contribuyentes. La falta de jurisprudencia y de pronunciamiento de las autoridades tributarias de los Países Miembros hace necesario que el medio académico retome el análisis de este artículo.

b) Es necesario explorar y darle mayor conte-

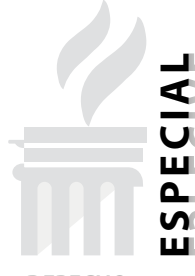

DERECHO TRIBUTARIO ADUANERO (1) 
nido a la relación existente entre el ordenamiento jurídico de la Comunidad Andina y la Decisión 578. Esta última suele revisarse de manera aislada y verse como "un convenio más" para evitar la doble imposición. Esto no es correcto.

c) De igual manera, es importante revisar el concepto de no-discriminación desarrollado por el TJCAN en otros casos diferentes al tributario y ver cómo los criterios de este tribunal pueden encajar en el planteamiento de una metodología para aplicar la cláusula de no-discriminación de la Decisión 578. d) Debe hacerse un llamado a la Comunidad Andina y las autoridades tributarias de sus Países Miembros para que, en aras de las libertades comunitarias, se establezcan guías y lineamientos no solo para la aplicación del artículo 18 de la Decisión 578, sino también de las otras disposiciones contenidas en esta Decisión.

e) Por último, la SUNAT debe implementar medidas administrativas que faciliten el control y la declaración de aquellos casos en los que resulta aplicable la cláusula de no-discriminación. 